\title{
LABORATÓRIOS REMOTOS DE FPGA COM FOCO NO ENSINO: UMA REVISÃO SISTEMÁTICA DA LITERATURA
}

\author{
Ana Lúcia da Silva Beraldo, ICT/Unifesp, ana.beraldo@unifesp.br \\ Tiago de Oliveira, ICT/Unifesp, tiago.oliveira@unifesp.br \\ Denise Stringini, ICT/Unifesp, dstringhini@unifesp.br
}

\begin{abstract}
Resumo. Esta revisão sistemática foi realizada para se ter uma visão geral do estado da arte sobre o desenvolvimento de laboratórios remotos específicos de FPGA (Field Programmable Gate Array) utilizados para o ensino de Circuitos Digitais ou disciplinas similares. Buscou-se identificar quais são as principais funcionalidades do sistema desenvolvido, se a ferramenta permite interação entre os usuários, se o sistema é livre ou comercial, quais equipamentos de hardware foram utilizados no projeto, se há integração com algum ambiente virtual de aprendizagem e se foram realizados testes do sistema com estudantes. Os resultados apontam a existência de um pequeno número de laboratórios remotos específicos de FPGA com foco no ensino sendo que, a maioria deles está concentrada na Europa.
\end{abstract}

Palavras-chave: laboratório remoto, FPGA, ensino, aprendizagem

\section{REMOTE FPGA LABORATORIES WITH FOCUS ON TEACHING: A SYSTEMATIC REVIEW OF LITERATURE}

\begin{abstract}
This systematic review was conducted to provide a state-of-the-art overview of the development of specific remote laboratories of FPGA (Field Programmable Gate Array) used for teaching Digital Circuits or similar subjects. We tried to identify the main functionalities of the developed system, whether the tool allows interaction between users and whether the system is free or commercial, which hardware equipment was used in the project; if the system permits integration with any virtual learning environment and if were carried out a test on the system with students. The results show us a small number of specific FPGA laboratories with a focus on teaching and the majority of which are concentrated in Europe.
\end{abstract}

Keywords: remote laboratory, FPGA, teaching, education

\section{Introdução}

Assim como lógica de programação é importante para o ensinamento do pensamento computacional, da formulação de um problema e de sua solução de forma que uma máquina possa realizá-lo, o ensinamento de sistemas digitais é importante para a compreensão e estabelecimento da lógica digital que rege o processo de funcionamento tecnológico de qualquer sistema computacional.

Sistemas Digitais constitui-se em uma temática extremamente relevante e importante em muitos cursos que envolvem tecnologia, principalmente nos currículos de cursos de graduação em Ciência da Computação e, especialmente, em Engenharia de Computação. Pode-se notar essa importância nos documentos referenciais orientadores produzidos por sociedades de computação internacionais e nacionais, como é o caso da ACM, IEEE e SBC. Especificamente para os cursos de graduação em Engenharia de Computação, conteúdos sobre Arquitetura e Organização de Computadores devem compor papel significante na formação acadêmica do aluno como relatado no currículo de referência da ACM/IEEE (CURRICULA, 2001). Neste documento, definem-se áreas de conhecimento que concentram determinados assuntos ou conteúdos específicos que envolvem computação e que devem ser abordados num currículo de Engenharia de 
Computação. Essas áreas de conhecimento são expressas em horas (core hours), correspondendo à quantidade mínima em sala de aula que esses conteúdos devem ser abordados. Neste contexto, a ACM/IEEE define treze áreas de conhecimento específico com as seguintes distribuições de horas: Circuitos e Eletrônicas (Circuits and Electronics) - 50 horas, Algoritmos Computacionais (Computing Algorithms) - 30 horas, Arquitetura e Organização de Computadores (Computer Architecture and Organization) - 60 horas, Projeto de Sistemas Digitais (Digital Design) - 50 horas, Sistemas Embarcados (Embedded Systems) - 40 horas, Redes de Computadores (Computer Networks) - 20 horas, Práticas Profissionais (Professional Practice) - 20 horas, Segurança da Informação (Information Security) - 20 horas, Estratégias para Tecnologias Emergentes (Strategies for Emerging Technologies) - 10 horas, Processamento de Sinal (Signal Processing) - 30 horas, Engenharia de Projetos e Sistemas (Systems and Project Engineering) - 30 horas, Gerenciamento de Recursos de Dados (Systems Resource Management) - 20 horas e, Projeto de Software (Software Design) - 40 horas. Considerando a relação intrínseca entre as duas áreas de conhecimento definidas como Arquitetura e Organização de Computadores e Projeto de Sistemas Digitais, perfazendo em conjunto 110 horas num total de 420 horas, pode-se perceber a grande importância dessa temática em um currículo de Engenharia de Computação.

Nacionalmente, a SBC também definiu um documento referente a um currículo de referência para cursos de graduação em Bacharelado em Ciência da Computação e Engenharia de Computação (CURRÍCULO, 2005). Nesse documento, a estruturação curricular ocorre por meio de núcleos de conhecimento, organizados da seguinte forma: Fundamentos da Computação, Tecnologia da Computação, Matemática, Ciências Básicas, Eletrônica e Contexto Social e Profissional. Conceitos fortemente relacionados com Arquitetura e Organização de Computadores são listados nos dois principais núcleos específicos da área de computação, a saber: Fundamentos da Computação (Circuitos Digitais e Arquitetura e Organização de Computadores) e Tecnologia da Computação (Sistemas Digitais). Mais recentemente, em 2012, a SBC e o Ministério da Educação (MEC) definiram algumas diretrizes curriculares para diversos cursos de graduação relacionados à área de computação (PARECER, 2012), discriminando conteúdos básicos e tecnológicos que devem ser escolhidos e trabalhados levando-se em consideração o perfil desejado do aluno egresso. Nessas diretrizes curriculares também pode-se notar a importância dessa temática, onde cinco de onze competências e habilidades específicas elencadas para os egressos de cursos de bacharelado em Engenharia de Computação referem-se a conceitos que estão diretamente relacionados com Sistemas Digitais, quais sejam: (1) Planejar, especificar, projetar, implementar, testar, verificar e validar sistemas de computação (sistemas digitais), incluindo computadores, sistemas baseados em microprocessadores, sistemas de comunicações e sistemas de automação, seguindo teorias, princípios, métodos, técnicas e procedimentos da Computação e da Engenharia; (2) Desenvolver processadores específicos, sistemas integrados e sistemas embarcados, incluindo o desenvolvimento de software para esses sistemas; (3) Analisar e avaliar arquiteturas de computadores, incluindo plataformas paralelas e distribuídas, como também desenvolver e otimizar software para elas; (4) Analisar, avaliar e selecionar plataformas de hardware e software adequados para suporte de aplicação e sistemas embarcados de tempo real; e (5) Analisar, avaliar, selecionar e configurar plataformas de hardware para o desenvolvimento e implementação de aplicações de software e serviços.

Sendo assim, devido a sua importância para os cursos de graduação em Computação, novas técnicas e metodologias para o ensino de sistemas digitais vêm sendo aplicadas em diversos currículos de Computação e publicadas na literatura científica nacional e internacional. Laboratórios remotos possibilitam que experimentos complexos 
ou que possuam equipamentos caros sejam compartilhados por meio da internet. Visando melhorar a oferta de disciplinas, as instituições de ensino que possuem equipamentos em número reduzido podem se beneficiar com este recurso.

Esta Revisão Sistemática da Literatura (RSL) visa, identificar na literatura, laboratórios remotos específicos de FPGA e responder à questão de pesquisa: "Quais são as principais características dos laboratórios remotos de FPGA dedicados às disciplinas de Sistemas Digitais?". O FPGA (Field Programmable Gate Array) é um circuito integrado produzido para ser reprogramado pelos usuários, ou seja, projetado para ser configurado após a fabricação. De acordo com Costa (2014), o FPGA é um dispositivo de alta complexidade que suporta a implementação de circuitos lógicos grandes e consiste em um grande arranjo de células lógicas ou blocos lógicos configuráveis contidos em um circuito integrado. É bastante utilizado nos cursos de Engenharia de Computação. Utilizado também para desenvolvimento de circuitos, testes e prototipação. Os Kits FPGA didáticos podem possuir muitos itens como, por exemplo, portas USB, interface de rede, interruptores, botões, display de 7 segmentos, display LCD, etc. Simplificando, o FPGA é um dispositivo físico (hardware) que permite aos estudantes a implementação de projetos de sistemas digitais em que os estudantes podem interagir por meio de seus componentes para enviar dados de entrada para o FPGA e visualizar os resultados obtidos nos sinais de saída. O Kit FPGA, por conter milhares de elementos lógicos, memória interna, memória flash, muitos conectores que possibilitam a comunicação com diferentes dispositivos, tem em seu preço um impeditivo para que os alunos possuam seus próprios Kits. Este valor também é impeditivo para que muitas universidades possam aumentar o número de laboratórios que oferecem esta tecnologia, pois, para a criação de um novo laboratório convencional, existe a necessidade de espaço físico, técnicos de laboratório para manter os equipamentos, computadores, mobiliário, entre outros. Isto justifica a importância da criação de laboratórios remotos.

O objetivo deste trabalho é identificar estudos relacionados ao desenvolvimento de laboratórios remotos específicos de FPGA. Os resultados obtidos da RSL realizada visam quais tecnologias têm sido aplicadas nos laboratórios remotos, quais as vantagens e desvantagens citadas pelos autores. Busca-se identificar ainda se os laboratórios permitem interação entre os usuários e se estão integrados a ambientes virtuais de aprendizagem.

\section{Material e métodos}

A RSL "consiste em uma forma de sintese dos resultados de pesquisas relacionados com um problema especifico" (GALVÃO; SAWADA; TREVISAN, 2009). Algumas das características da RSL, indicadas por Kitchenham e Charters (2007) são: a definição da questão de pesquisa e os métodos que serão utilizados para conduzir a RSL, documentação da estratégia realizada na pesquisa para que a mesma possa ser replicada, definição de critérios de avaliação para inclusão e exclusão dos estudos, dentre outros. Tais critérios são utilizados, de acordo com Galvão, Sawada e Trevisan (2009), para se evitar o viés no resultado da pesquisa científica proporcionando rigor em todas as fases do trabalho, de forma que possa ser reproduzida por outro autor e que se possa encontrar os mesmos resultados. Todos estes processos são seguidos para que ao término se obtenha exatidão nos dados obtidos.

A metodologia adotada para esta revisão sistemática é a descrita pelos autores Ferenhof e Fernandes (2016), que propõe o método Systematic Search Flow - SSF, composto por quatro fases: 
Fase 1-Protocolo: Nesta fase inicial é definida a estratégia de busca, são definidas a String de busca e as bases de dados que serão pesquisadas, a bibliografia encontrada é organizada por meio de algum software que automatize esta tarefa, é realizada a seleção dos artigos pela leitura do título, abstract e palavras-chave já aplicando os critérios de inclusão e exclusão. E finalmente é realizada a leitura dos artigos selecionados na íntegra.

Fase 2-Análise dos dados: É a fase em que o autor faz a combinação dos dados e dá início à análise dos resultados encontrados.

Fase 3-Síntese: $O$ autor retira as conclusões sobre o assunto estudado.

Fase 4-Escrita: Esta fase destina-se à escrita do artigo baseando-se nas informações que foram consolidadas na fase de síntese e são respondidas as questões de pesquisa.

Nas próximas subseções são evidenciadas as questões de pesquisa, define-se a String de busca, descrevem-se as técnicas que são utilizadas para avaliar os artigos no processo de inclusão e exclusão na revisão sistemática e apresenta-se a matriz de conhecimento que traz as questões utilizadas para realizar a extração dos dados.

\subsection{Protocolo da pesquisa}

Tema: Laboratórios remotos específicos de FPGA.

Objetivo: O principal objetivo desta RSL é fornecer uma visão geral dos trabalhos que tratam de laboratórios remotos de FPGA destinados ao ensino de Sistemas Digitais ou disciplinas equivalentes.

Questão principal: Quais são as principais características dos laboratórios remotos de FPGA dedicados ao ensino de Sistemas Digitais?

\section{Definição da String de busca e das bases de dados}

A estratégia de busca está separada em 3 partes: definição das palavras-chave, definição da String de busca e definição das bases de dados.

As palavras-chave definidas para o trabalho são:

- Remote laboratory

- FPGA

A String de busca foi construída combinando-se as palavras-chave e utilizando o operador lógico "AND":

((“remote laboratory") AND ("FPGA"))

As bases de dados selecionadas para este trabalho são as que tem alta relevância para o tema em estudo: IEEE, ACM, SpringerLink e Science Direct.

Esta RSL buscou, nas bases de dados, conteúdo que tivesse relação com laboratórios remotos de FPGA em publicações estrangeiras sem limitação por data.

\section{Matriz do conhecimento}

De acordo com Ferenhof e Fernandes (2016), a matriz de conhecimento é formada pelas questões que se deseja responder quando se faz uma revisão sistemática. No entanto, nem todas as questões podem ser respondidas em todos os artigos.

A matriz de conhecimento desta revisão busca responder questões direcionadas ao contexto de laboratórios remotos específicos de FPGA. A matriz de conhecimento, apresentada na Figura 1 traz as questões que se busca esclarecer. 


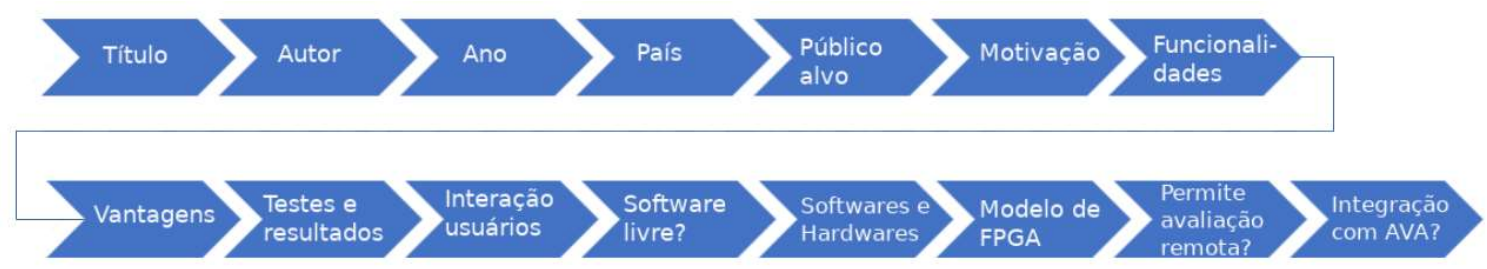

Figura 1 - Matriz de conhecimento desta Revisão Sistemática

\section{Critérios de seleção dos artigos}

A inclusão de critérios para a seleção dos artigos ajuda a filtrar os documentos relevantes daqueles que não tem ligação com o tema proposto. A Tabela 1 apresenta os critérios de inclusão e exclusão utilizados nesta revisão sistemática.

Tabela 1 - Critérios de inclusão e exclusão

\begin{tabular}{|c|c|}
\hline Critérios de INCLUSÃO & Critérios de EXCLUSÃO \\
\hline $\begin{array}{l}\text { Artigos que descrevam a utilização de } \\
\text { laboratório remoto de FPGA. }\end{array}$ & $\begin{array}{l}\text { Artigos que descrevam outros tipos de } \\
\text { laboratório remoto. }\end{array}$ \\
\hline $\begin{array}{l}\text { Artigos publicados em conferências ou } \\
\text { revistas científicas. }\end{array}$ & $\begin{array}{l}\text { Artigos que não estejam relacionados com as } \\
\text { questões de pesquisa. }\end{array}$ \\
\hline $\begin{array}{l}\text { Artigos que estiverem escritos no idioma } \\
\text { português ou inglês. }\end{array}$ & $\begin{array}{l}\text { Artigos escritos em outros idiomas além de } \\
\text { português ou inglês. }\end{array}$ \\
\hline
\end{tabular}

\section{Obtenção dos artigos}

O primeiro passo foi realizar a busca nas bases de dados selecionadas utilizando a String de busca. Os últimos acessos às bases de dados foram realizados na data de 19 de agosto de 2019 e os resultados estão listados na Tabela 2.

\begin{tabular}{l|c}
$\begin{array}{l}\text { Tabela } 2-\text { Resultados obtidos nas bases de dados selecionadas } \\
\text { Base Bibliográfica }\end{array}$ \\
\hline Número de artigos
\end{tabular}

Todos os 75 artigos foram analisados inicialmente pela leitura do título, abstract e foi verificado se atendiam à questão principal de pesquisa que é de ser um laboratório remoto de FPGA destinado ao ensino de sistemas digitais ou disciplinas similares, nesta seleção já foram aplicados os critérios de inclusão e exclusão. Após esta filtragem e removendo as duplicidades sobraram 23 artigos que descrevem especificamente o desenvolvimento de laboratórios remotos de FPGA relacionados com disciplinas de sistemas digitais ou disciplinas similares. Estes artigos foram lidos na íntegra e foi realizada a extração dos dados que permitiram responder à questão de pesquisa proposta. As referências completas dos 23 artigos utilizados nesta RSL são listadas no link https://tinyurl.com/s2ncvom.

$\mathrm{Na}$ listagem de referências bibliográficas consta, para cada artigo, um identificador [ID] o qual será utilizado nas próximas subseções para citar os artigos identificados durante a extração dos dados desta RSL. 


\section{Resultados e discussão}

Os artigos selecionados para esta RSL foram publicados entre os anos de 2004 a 2018, porém não havia critério de exclusão quanto a data de publicação no protocolo da RSL. Os anos em que houve maior número de publicações foram em 2014 e 2018 com um total de 4 artigos em cada ano.

Os primeiros itens analisados foram o continente e país em que o laboratório foi desenvolvido. E, como se pode observar na Figura 2, o continente em que houve o maior número de publicações foi o Europeu com um total de 12 artigos [ID 1, 4, 7, 8, 10, 11, 13, 14, 15, 17, 21 e 23], seguido do Americano com 6 [ID 5, 6, 18, 19, 20 e 22], Asiático com 4 [ID 2, 9, 12 e 16] e Africano com uma publicação [ID 3]. Os países que tiveram o número maior de publicações individualmente foram Espanha e Estados Unidos totalizando 4 estudos cada um.

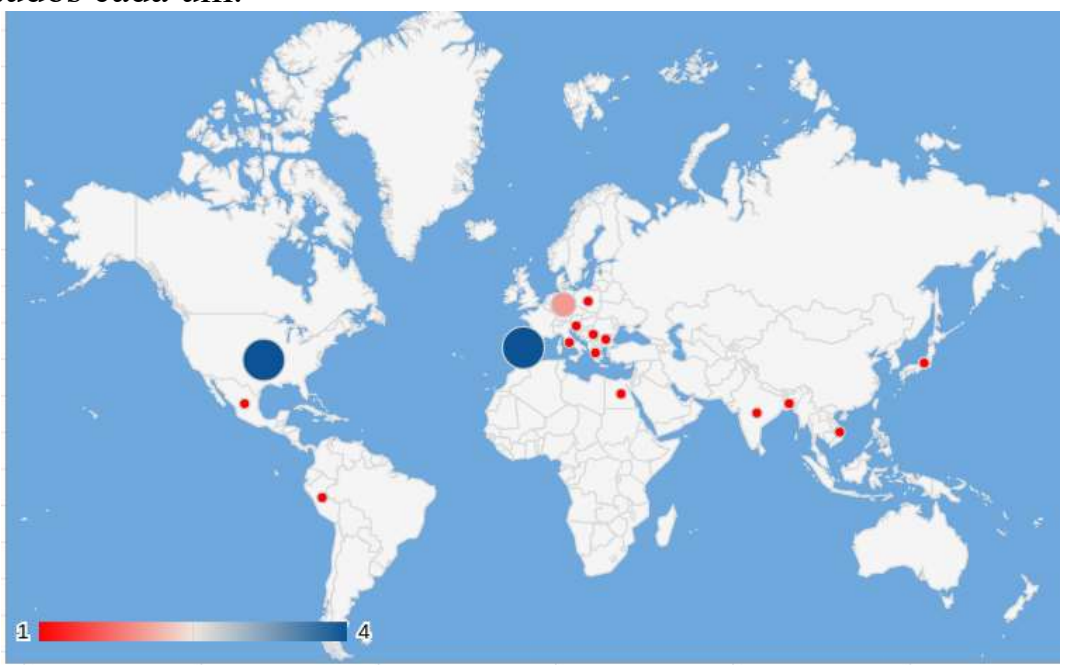

Figura 2 - Regiões de incidência dos laboratórios remotos de FPGA

Devido às especificidades dos Kits FPGA, dos diversos modelos disponíveis e dos outros componentes que podem ser conectados a eles, cada um dos estudos propõem soluções bem distintas para o desenvolvimento do laboratório remoto de FPGA. E apenas dois deles disponibilizam o seu sistema como software livre [ID 21 e 22].

O público-alvo dos laboratórios desenvolvidos está dividido em nível superior [ID 1, 4, 5, 9, 10, 11, 12, 13, 14, 16, 18 e 21]; ensino a distância [ID 3], sendo este, um laboratório remoto de FPGA adequado para cursos completos de e-learning em design de hardware de um curso de Engenharia da Computação; e ainda, há um laboratório destinado a treinamentos e aplicações industriais [ID 7], tratando-se de um laboratório de prototipagem remota que suporta o design de sistemas eletrônicos. Alguns dos artigos não deixaram explícito o público para o qual o seu laboratório foi desenvolvido [ID 2, 6, 8, $15,17,19,20,22$ e 23$]$.

Nem todos os estudos citam suas motivações, vantagens ou problemas enfrentados durante o projeto. Ainda assim, algumas das motivações para desenvolver o laboratório remoto podem ser observadas na Tabela 3 .

\section{Tabela 3 - Motivações identificadas nos artigos}

\begin{tabular}{l|l} 
Motivações & ID \\
\hline Complementar e apoiar as aulas. & {$[1$ e 2] } \\
\hline Limitação de recursos de laboratório técnicos e humanos. & {$[10]$} \\
\hline
\end{tabular}


Suprir carência de laboratório prático.

Maior mobilidade, menos riscos de segurança.

Tempo e espaço de laboratório restritos.

$[22]$

As funcionalidades do sistema são tarefas que o usuário poderá executar ao utilizar um sistema. Buscamos saber quais são as principais funcionalidades que estes sistemas disponibilizam aos alunos e professores e as mesmas são destacadas na Tabela 4.

Tabela 4 - Funcionalidades existentes nos laboratórios remotos

\begin{tabular}{l|l} 
Funcionalidades & ID \\
\hline $\begin{array}{l}\text { O sistema faz a correção automática dos trabalhos baseado em expressões } \\
\text { regulares, o sistema testa a solução do aluno baseado em um arquivo } \\
\text { padrão do professor. }\end{array}$ & {$[11]$} \\
\hline $\begin{array}{l}\text { Compartilhamento de resultados e materiais dos estudantes. } \\
\text { Serve para múltiplos laboratórios. }\end{array}$ & {$[12]$} \\
\hline $\begin{array}{l}\text { Suporta grupos de até 5 pessoas atuando na mesma atividade, permite } \\
\text { trabalho colaborativo. }\end{array}$ & {$[5]$} \\
\hline $\begin{array}{l}\text { Apresenta os resultados da interação com o FPGA sem a necessidade de } \\
\text { webcam. }\end{array}$ & $\begin{array}{l}{[6,9,11,} \\
16,18 \text { e 23] }\end{array}$ \\
\hline
\end{tabular}

Algumas das vantagens, decorrentes da implantação do laboratório remoto e listadas pelos autores, podem ser observadas na Tabela 5.

Tabela 5: Vantagens e desvantagens identificadas nos artigos

Vantagens

Poder ter turmas maiores com menor custo mínimo uso de banda de internet e [18] compartilhamento de recursos.

Mínimo uso de banda de internet e compartilhamento de recursos de hardware.

Realizar o apoio à prototipagem sucessiva de módulos de um sistema e fazer a [7] validação dos protótipos simulando o cenário de implantação.

Acessibilidade e escalabilidade.

Desvantagens

Não há interface gráfica no sistema desenvolvido, tudo é feito por linha de [19] comando.

Quanto a realização de testes e avaliação do sistema desenvolvido, em 9 dos trabalhos [ID 1, 4, 5, 6, 7, 11, 13, 14 e 19] foi apresentado algum tipo de feedback dos alunos com relação a sua experiência quanto ao uso dos laboratórios por meio de questionários de avaliação aplicados após a utilização do laboratório remoto. A Tabela 6 apresenta alguns dos resultados obtidos.

Tabela 6: Resultados dos laboratórios remotos

\section{Resultado/Feedbacks}

Os estudantes aceitaram bem a combinação dos sistemas local e remoto.

O curso foi muito bem-sucedido entre os alunos, com $73 \%$ deles descrevendo-o como excelente e $27 \%$ como uma experiência muito boa. 
A maioria dos alunos (88\%) afirmou que esse ambiente os ajudou a obter conhecimentos mais práticos. A maioria dos alunos (90\%) apreciou a maneira fácil de se conectar ao sistema.

Não foram recebidos muitos feedbacks positivos pois os menus de ajuda e [6] tutoriais não existiam como instruções básicas de acesso ao sistema.

Todos apreciaram a simplicidade da configuração, a atraente relação custobenefício e a liberdade de acessar os laboratórios a qualquer hora e em qualquer lugar.

Os alunos declararam que é possível usar o laboratório remoto sem a necessidade de trabalhar com o equipamento real. E, quanto à interface gráfica do usuário, afirmam que é fácil de usar e que são capazes de aprender rapidamente.

A iniciativa foi bem aceita pelos alunos, que deram o valor de positivo ou muito positivo à existência do laboratório remoto. No final das atividades, os alunos ficaram muito interessados em incluir essa ferramenta em cursos futuros.

A experiência foi muito popular entre os alunos e o resultado desejado foi alcançado.

A resposta dos alunos aos laboratórios remotos em geral foi positiva. Os alunos gostaram da flexibilidade de trabalhar de qualquer lugar no campus e a qualquer momento e também se sentiram livres para levar mais tempo para explorar os exercícios. Os alunos não responderam bem à falta de feedback físico e visual do sistema.

Outra questão que se buscava responder era se os laboratórios utilizavam uma webcam para mostrar seus resultados ou se havia sido desenvolvido algum outro meio de apresentar os resultados do FPGA na tela dos usuários. Os trabalhos [ID 6, 9, 11, 16, 18 e 23] afirmaram não utilizar a webcam, tendo para isto, desenvolvido alguma funcionalidade que apresenta os resultados do FPGA na tela do usuário. Observou-se que a solução tomada por alguns dos autores foi a de fazer a transmissão dos resultados apresentados no Kit FPGA pela porta de comunicação RS232 do Kit FPGA, pois, de acordo com [16] possibilita uma velocidade rápida de tráfego dos dados sem comprometer a banda de internet. Os trabalhos [ID 7, 10, 12, 15, 21 e 22] utilizam exclusivamente a webcam como opção de visualização dos resultados. E os trabalhos [ID $1,2,3,4,5,8,13,14,17,19$ e 20] não explicitaram como é realizada a apresentação de resultados em seu laboratório remoto. Este item é importante pois, dependendo da velocidade da internet do estudante, a atividade pode apresentar erros por causa da lentidão. Sendo assim, quanto mais eficiente e rápida a troca de dados entre cliente e servidor, melhor será a resposta ao usuário.

Quanto à possibilidade de colaboração nono ambiente web de um sistema digital por meio de um laboratório remoto, foi verificado que em apenas um estudo [ID 5] os alunos podem fazer o login simultaneamente e interagir colaborativamente durante a realização das atividades.

As principais fabricantes de FPGA são a Xilinx e a Altera. A Xilinx, fundada em 1984 na Califórnia, é a primeira inventora do FPGA e primeira empresa de semicondutores a se especializar na fabricação de hardware. Atualmente é a maior fornecedora do mundo de dispositivos lógicos programáveis. Por sua vez, a Altera, uma empresa fabricante de dispositivos lógicos programáveis. Ela, foi fundada em 1984 e foi comprada pela Intel Corporation em 2015. Seus principais produtos são as FPGAs Stratix, Arria e Cyclone e o software Quartus Prime. Quanto ao kit de FPGA utilizado nos laboratórios remotos, a maioria utiliza vários modelos do FPGA da Xilinx [3, 5, 7, 8, 10 , $11,13,17,18,19,21$ e 22] e em menor número os modelos da Altera/Intel [ID 2, 4, 9, V. $18 \mathrm{~N}^{\circ} 1$, julho, 2020 RENOTE DOI: 
12, 12 e 20]. Alguns dos trabalhos, no entanto, não descreveram qual foi o modelo de FPGA utilizado no projeto.

Uma linguagem de descrição de hardware (Hardware Description Language HDL) pode ser utilizada para descrever o funcionamento de um sistema digital, a sua concepção e organização. Uma HDL pode também testar o hardware para verificar seu funcionamento por meio de simulação. Um sistema descrito em linguagem de hardware pode ser implementado em um dispositivo programável como o FPGA. As linguagens de descrição de hardware utilizadas para a realização das atividades nos laboratórios remotos encontrados são VHDL [ID 4, 7, 11, 13, 14, 15, 18, 21 e 23], Verilog [ID 9, 12 e 18] e em 11 estudos a linguagem utilizada não foi descrita [ID 1, 2, 3, 5, 6, 8, 10, 16, $17,19,20$ e 22$]$.

E, finalmente, buscávamos saber quanto a integração do laboratório com um ambiente virtual de aprendizagem. Foi identificado em apenas três estudos [ID 4, 11, 17] o acesso ao laboratório remoto por meio de um AVA, sendo que em apenas um deles se utiliza o Moodle e, nos outros dois se utiliza outros sistemas proprietários. Nos outros trabalhos o acesso ao laboratório remoto é feito diretamente pelo sistema do laboratório remoto, não possuindo integração com nenhum ambiente virtual de aprendizagem.

\section{Conclusões}

Diante das respostas obtidas nesta RSL retomamos a questão de pesquisa "Quais são as principais características dos laboratórios remotos de FPGA dedicados ao ensino de Sistemas Digitais?", e podemos respondê-la da seguinte forma: foi observado que as pesquisas sobre laboratórios remotos exclusivos de FPGA não são em grande número e estão sendo realizadas em países estrangeiros. A Europa é onde mais se pesquisa sobre laboratórios remotos de FPGA. Os cursos onde se aplicam os laboratórios são basicamente os de exatas como as Engenharias, Mecatrônica e Computação. A implantação dos laboratórios tem ocorrido para acompanhar a evolução da tecnologia, para motivar os alunos a estudar, para oferecer outras formas de acesso ao sistema, devido a melhor flexibilidade e acessibilidade, para promover um melhor uso dos equipamentos e também por razões de espaço físico e questões financeiras.

Os Kits FPGA mais utilizados são os das fabricantes Xilinx (em maior número) e seguidos pela Altera/Intel. E as linguagens de descrição de hardware utilizadas são VHDL (em maior número) seguida pela linguagem Verilog. Alguns dos laboratórios possuem funcionalidades interessantes como ter a correção automática das atividades baseada em um arquivo padrão, manter a interação entre os alunos e apresentar os resultados do FPGA de outras formas que não sejam com a webcam. Os testes e pesquisas realizados com os alunos indicaram que o laboratório remoto tem sido bem aceito e que os alunos manifestam o interesse em manter os laboratórios em funcionamento.

Por fim, vale ressaltar que a maioria dos softwares destinados aos laboratórios remotos de FPGA não estão disponíveis para download e o seu uso ocorre de forma proprietária não gratuita ou extremamente restrita, dificultando ou impossibilitando a difusão desses laboratórios para outros contextos e realidades educacionais. Deve-se trabalhar o uso de software livre e o desenvolvimento de laboratórios remotos de FPGA abertos para que todos possam usufruir dos benefícios desse sistema, com vistas a intensificar uma cultura de pesquisa e projeto na área de sistemas digitais bem como na área de desenvolvimento de soluções em FPGA, disseminando-os, principalmente, em territórios que possuem pouca infraestrutura ou recursos financeiros.

\section{Referências}


ALI, Md Liakot; RAHMAN, Md Habibur; RAHAMAN, Md Abu Nayeem Redwanur. Development of a remote digital system laboratory. In: 2012 15th International Conference on Computer and Information Technology (ICCIT). IEEE, 2012. p. 575580.

AMARAL, Érico et al. Laboratório virtual de aprendizagem: uma proposta taxonômica. RENOTE, v. 9, n. 2, 2011.

BRITO, Lélis Maia de et al. Ambientes virtuais de aprendizagem como ferramentas de apoio em cursos presenciais e a distância. RENOTE, v.11, n.1, 2013.

CALDEIRA, Ana Cristina Muscas. Avaliação da aprendizagem em meios digitais: novos contextos. In: XI Congresso Internacional de Educação a Distância. 2004.

COSTA, C. Projetos de Circuitos Digitais com FPGA. [S.1.: s.n.], 2014. ISBN 978-85365-0585-5.

CURRICULA, Computing. The Joint Task Force on Computing Curricula IEEE Computer Society and the Association for Computing Machinery. 2001.

CURRÍCULO de referência da SBC para cursos de Graduação em Bacharelado em Ciência da Computação e Engenharia de Computação. SBC - Sociedade Brasileira de Computação. 2005.

DE FREITAS, Alfredo Américo; BAUCHSPIESS, Adolfo; BORGES, Geovany Araújo. Laboratório de ensino de automação remoto da UnB. In: Anais: Congresso Brasileiro de Ensino de Engenharia. Brasília. 2004.

DE SOUZA, Antonio Lopes; DE OLIVEIRA, José Carlos; DE LIMA SANTOS, Marcelo Paulino. Recursos da computação gráfica para o desenvolvimento de um laboratório virtual de Teoria Eletromagnética. In: Anais do XXIX Congresso Brasileiro de Ensino de Engenharia, Porto Alegre, Setembro de. 2001.

DUARTE MEDINA, Roseclea; TAROUCO, Liane Margarida Rockenbach; AMORETTI, Suzana. Laboratório Virtual ASTERIX-resultados decorrentes da sua utilização como ferramenta cognitiva. In: X Congreso Argentino de Ciencias de la Computación. 2004.

FERENHOF, Helio Aisenberg; FERNANDES, Roberto Fabiano. Desmistificando a revisão de literatura como base para redação científica: método SSF. Revista ACB, v. 21, n. 3, p. 550-563, 2016.

FROZZA, Rejane et al. Agentes pedagógicos emocionais atuando em um ambiente virtual de aprendizagem. RENOTE, v. 9, n. 1, 2011.

GALVÃO, C. M.; SAWADA, N. O.; TREVISAN, M. A. Revisão sistemática: recurso que proporciona a incorporação das evidências na prática da enfermagem. Rev Lat Am Enferm. 2004; 12 (3): 549-56. Rev Esc Enferm USP, v. 43, n. 2, p. 465-71, 2009.

GOMES, Luis et al. Remote experimentation for introductory digital logic course. In: 20093rd IEEE International Conference on E-Learning in Industrial Electronics (ICELIE). IEEE, 2009. p. 98-103.

GUAITA, Renata Isabelle; GONÇALVES, Fábio Peres. A experimentação na educação à distância: reflexões para a formação de professores de ciências da natureza. In: XI Congresso Brasileiro do Ensino Superior à Distância. 2014. p. 1461-1475.

INDRUSIAK, Leandro Soares; GLESNER, Manfred; REIS, Ricardo. On the evolution of remote laboratories for prototyping digital electronic systems. IEEE Transactions on Industrial Electronics, v. 54, n. 6, p. 3069-3077, 2007.

KITCHENHAM, Barbara; CHARTERS, Stuart. Guidelines for performing systematic literature reviews in software engineering. EBSE Technical Report. 2007. Disponível em: $<$ https://elsevier.com/_data/promis_misc/525444systematicreviewsguide.pdf $>$. Acesso em: 16 de set. 2019. 
PARECER CNE/CES n. 136, de 09 de março de 2012, que trata sobre as Diretrizes Curriculares Nacionais para os cursos de graduação em Computação.

PERSIANO, Giovanni Vito et al. Distance learning in digital electronics: Laboratory practice on FPGA. In: 2007 IEEE Instrumentation \& Measurement Technology Conference IMTC 2007. IEEE, 2007. p. 1-6.

RAJASEKHAR, Yamuna et al. Teaching FPGA system design via a remote laboratory facility. In: 2008 International Conference on Field Programmable Logic and Applications. IEEE, 2008. p. 687-690.

ZUBIA, J.-G. Programmable Logic and Weblabs. In: Microelectronics Education. Springer, Dordrecht, 2004. p. 277-281. 